

\title{
UMA METODOLOGIA DE APOIO À TOMADA DE DECISÃO PARA A SELEÇÃO DE MATERIAIS NO DESENVOLVIMENTO DE PROJETOS DE CADEIRAS DE RODAS
}

\author{
A DECISION SUPPORT METHODOLOGY FOR MATERIAL SELECTION IN THE \\ DEVELOPMENT OF WHEELCHAIR PROJECTS
}

\author{
(iD) Marcia Danieli Szeremeta Spak ${ }^{1}$ \\ iD Aldo Braghini Junior ${ }^{2}$ \\ D Joao Carlos Colmenero ${ }^{3}$
}

${ }^{1}$ Doutora em Engenharia de Produção Universidade Tecnológica Federal do Paraná - UTFPR. Pato Branco, Paraná - Brasil. spak@utfpr.edu.br

2 Doutor em Engenharia Mecânica Universidade Tecnológica Federal do Paraná - UTFPR. Ponta Grossa, Paraná - Brasil. aldo@utfpr.edu.br

${ }^{3}$ Doutor em Ciência e Engenharia dos Materiais Universidade Tecnológica Federal do Paraná - UTFPR. Ponta Grossa, Paraná - Brasil. colmenero@utfpr.edu.br

Recebido em: 29 abr. 2020

Aprovado em: 04 jul. 2020
Resumo: Uma das principais etapas de um projeto de cadeira de rodas é a definição de sua estrutura, que deve ser resistente para possibilitar a sua utilização em vários ambientes, leve para evitar a fadiga e possíveis problemas de saúde nos membros superiores do usuário, e possuir um custo adequado. O objetivo deste artigo é propor um método para a seleção do material da estrutura da cadeira de rodas de modo a auxiliar na etapa de desenvolvimento e melhoria de projetos de cadeiras de rodas. Para isso, foi empregado o método multicritério de tomada de decisão Analytic Hierarchy Process (AHP), o qual considera critérios quantitativos e qualitativos predeterminados para classificar as alternativas analisadas. O método foi aplicado para a seleção do material da estrutura de uma cadeira de rodas considerando dez alternativas (aços, ligas de alumínio e titânio). Nessa análise, identificou-se que a liga de alumínio A7003-T5, é o melhor material para a estrutura de cadeiras de rodas.

Palavras-chave: Tomada de decisão. AHP. Seleção de materiais. Cadeira de rodas.

Abstract: One of the main stages of a wheelchair project is the definition of the structure that must be resistant to allow its use in various environments, light to avoid fatigue and possible health problems in the user's upper limbs and have an adequate cost. The objective of this article is to propose a methodology for the material selection of wheelchair structure to assist in the development and improvement of wheelchair projects. For that, the Analytic Hierarchy Process (AHP) multicriteria decision-making method was used, which considers predetermined quantitative and qualitative criteria to classify the analyzed alternatives. The method was applied to material selection of a wheelchair structure considering ten alternatives (steels, aluminum alloys and titanium). In this analysis it was identified that the aluminum alloy A7003-T5, is the best material for the wheelchair structure.

Keywords: Decision-making. AHP. Material selection. Wheelchair. 


\section{Introdução}

Cadeiras de rodas são dispositivos de assistência fundamentais para os indivíduos que apresentam restrições de locomoção definitiva ou temporária nos membros inferiores causadas por doenças, traumatismos ou outras condições incapacitantes. Em torno de 10\% da população global possui algum tipo de deficiência, e cerca de 10\% dessa população com deficiência, é usuária de cadeira de rodas (Mistarihi, Okour, \& Mumani, 2020). A cadeira de rodas é uma tecnologia assistiva, considerada um equipamento médico durável, que deve atender as necessidades de conforto, mobilidade, independência e segurança dos usuários, e também possuir qualidade, sendo capaz de suportar o uso repetitivo com o mínimo de reparos (Yuan \& Guan, 2014; Fitzgerald, Cooper, Boninger, \& Rentschler, 2001)

O ambiente físico e as características tecnológicas das cadeiras de rodas (tipos de dispositivo e modo de utilização) são os principais fatores limitantes para o convívio social e para a mobilidade do usuário (Ravenek, Ravenek, Hitzig, \& Wolfe, 2012; Koontz, Brindle, Kankipati, Feathers, \& Cooper, 2010). Cadeiras de rodas inadequadas interferem na independência do usuário, acarretando em dificuldade de movimentação e contribuindo para a realização de esforços repetitivos que acabam levando o usuário à fadiga, dores e lesões nos membros superiores (Mistarihi et al., 2020; Hybois et al., 2019; Ahmad, Maidin, Rahman, \& Osman, 2017). Quando a cadeira de rodas é adequada as necessidades de mobilidade e conforto, os usuários tendem a utilizá-la com mais facilidade e independência. Cadeiras de rodas mais eficientes podem permitir que a capacidade funcional seja elevada, exigindo menor esforço do usuário (Sprigle, 2009). Ter o dispositivo de mobilidade adequado possibilita a melhoria do convívio social do usuário, tornando-o mais ativo e independente (Hybois et al., 2019; Ward et al., 2010).

Um fator importante para a independência do usuário está relacionado as tecnologias adotadas que facilitam a sua mobilidade. Segundo Koontz et al. (2010) a tecnologia deficitária das cadeiras de rodas, especificamente o peso, é um dos principais problemas relacionados a mobilidade relatados pelos usuários de cadeiras de rodas. As cadeiras de rodas desenvolvidas com materiais de baixo custo, geralmente possuem baixa qualidade e durabilidade (Yuan \& Guan, 2014). Também são de difícil manuseio devido ao peso, exigindo assim um maior esforço do usuário para a sua locomoção.

Buscando solucionar esses problemas, pesquisas na área de engenharia vêm sendo desenvolvidas visando aprimoramentos e melhorias em diversos aspectos das cadeiras de rodas. Estudos como o de Mistarihi et al. (2020) propuseram um novo design de cadeira de rodas, buscando evitar problemas e lesões relacionadas às posturas corporais tanto do usuário, como do acompanhante. Ahmad et al. (2017) buscaram selecionar o melhor conceito de design de cadeira de rodas manual visando atender as necessidades de idosos. Ruíz-Serrano et al. (2013) desenvolveram um projeto de cadeira de rodas inteligente por meio de um sistema que utiliza controle de expressão e controle 
magnético para a direção da cadeira de rodas, destinada a pacientes com deficiência motora grave. Berger, Van Nieuwenhuizen, Van Der Ent, \& Van Der Zande (2012) propuseram o desenvolvimento de um projeto de cadeira de rodas voltada a prática de esportiva por meio da redução do peso da cadeira de rodas e adaptação as medidas antropométricas dos esportistas. Quaglia, Franco, \& Oderio (2011) propuseram um projeto de cadeira de rodas motorizada que possa ultrapassar obstáculos e subir escadas.

Vários materiais podem ser utilizados para a fabricação de cadeiras de rodas. A escolha do material adequado possibilita a personalização e o ajuste às necessidades do usuário, visando o maior conforto, melhor performance e facilidade de uso da cadeira de rodas (Koontz et al., 2010). Existem vários métodos que podem ser utilizados para a seleção de materiais que variam conforme o contexto abordado. Tendo em vista a interligação dos aspectos técnicos dos materiais com o custo, e os aspectos subjetivos relacionados à decisão, a utilização de uma metodologia de tomada de decisão multicritério torna-se adequada.

Os métodos multicritérios consideram todas as variáveis importantes do processo decisório, agregando informações quantitativas e qualitativas à tomada de decisão, identificando os atores de influência e as alternativas viáveis, buscando a coerência entre o objetivo inicial e os resultados (Ahmad et al., 2017; Roy \& Vanderpooten, 1996; Banville, Landry, Martel, \& Boulaire, 1998).

Um dos principais métodos multicritérios é o Analytic Hierarchy Process (AHP). O método AHP realiza uma representação hierárquica dos elementos envolvidos no processo, para a melhor visualização do contexto de tomada de decisão. É uma técnica que apoia os tomadores de decisão na estruturação de decisões complexas, quantificando fatores intangíveis e avaliando escolhas em situações de decisão com vários cenários (Ahmad et al., 2017). O método AHP é aplicado em vários cenários de decisão. Mohammadshahi (2013) e Vaidya e Kumar (2006) apresentaram diferentes aplicações do método AHP que envolvem desde áreas de cunho pessoal até variações entre os campos da medicina, administração, política, engenharia e esportes.

Métodos multicritério de tomada de decisão, entre ele o método AHP, foram utilizados em projetos de cadeiras de rodas. Mistarihi et al. (2020) aplicaram os métodos Quality Function Deployment (QFD) e Fuzzy Analytic Network Process (FANP) para a melhoria do encosto e apoio das mãos da cadeira de rodas. Ahmad et al. (2017) aplicaram o método AHP para selecionar o melhor projeto de cadeira de rodas manuais para idosos. Nwaoha e Ashiedu (2015) aplicaram o método AHP para selecionar os fatores mais importantes a serem contemplados em um projeto de cadeira de rodas. Yuan e Guan (2014) aplicaram o método AHP em conjunto com o modelo Kano para o desenvolvimento de projetos de cadeira de rodas individualizadas, que atendam aos requisitos funcionais dos usuários.

A utilização do método AHP para a seleção de materiais para a confecção de estruturas de cadeiras de rodas, visa auxiliar as instituições promotoras de pesquisas assistivas no desenvolvimento 
de projetos voltados ao aprimoramento das tecnologias e melhorias no desempenho das cadeiras de rodas.

Embora cada projeto tenha um objetivo e função individual, a estrutura da cadeira de rodas deve ser adaptada aos vários contextos, tornando-se necessária a análise do material empregado para atender as especificações de cada projeto em relação a densidade, resistência, processamento e custo do material empregado para a confecção da estrutura da cadeira de rodas. Sendo assim, o presente artigo tem o objetivo de identificar o melhor material para a fabricação de estruturas de cadeiras de rodas entre as alternativas analisadas e considerando os critérios adotados para a análise, por meio da aplicação do método AHP.

\section{Metodologia}

A metodologia será desenvolvida para a seleção do melhor material para a confecção de estruturas de cadeiras de rodas. Existem vários tipos e modelos de cadeiras de rodas, de maneira genérica as principais são: as motorizadas, manuais e esportivas. A proposta do estudo não visa um modelo em específico, mas sim ao auxílio no desenvolvimento de projetos voltados ao aprimoramento dos dispositivos de acordo com as necessidades do usuário.

O método AHP é empregado para essa seleção, fornecendo o vetor de prioridade para os materiais analisados. A abordagem do método AHP consiste na definição do problema ou objetivo, identificação dos critérios e subcritérios que influenciam a tomada de decisão, identificação das alternativas que permitam atingir o objetivo, aplicação da comparação de pares entre os critérios, subcritérios e alternativas para a definição das prioridades, cálculo dos índices de consistência e a síntese de prioridades (Vaidya \& Kumar, 2006; Çaliskan, Kursuncu, Kurbanoglu, \& Güven, 2013; Ahmad et al., 2017). O método tem sido amplamente utilizado em vários contextos de decisões complexas, permite aos decisores: modelar um problema por meio de uma estrutura hierárquica que mostra a relação entre o objetivo, os critérios, subcritérios e as alternativas; integrar a informação à experiência; e medir a importância relativa através da comparação por pares (Arunraj \& Maiti, 2010).

O método AHP é desenvolvido através dos passos a seguir.

\subsection{Desenvolvimento da estrutura hierárquica}

A premissa principal do método AHP é decompor o problema de decisão em níveis hierárquicos, facilitando a análise e a compreensão da tomada de decisão (Mayyas et al., 2011). A estruturação do problema de decisão em níveis hierárquicos possui dois propósitos: fornecer uma visão geral da complexa relação das variáveis inerentes ao problema e ajudar o decisor a fazer o julgamento correto na comparação de elementos que estão no mesmo nível hierárquico (Arunraj \& Maiti, 2010). O primeiro 
passo para a aplicação do método consiste na definição do objetivo da análise, seguido da identificação dos critérios adequados para a tomada de decisão, e das alternativas que responderam o objetivo inicial.

No presente estudo, o método AHP é constituído por uma estrutura hierárquica de três níveis, o primeiro nível é composto pelo o objetivo: a seleção do melhor material para a fabricação de estruturas de cadeira de rodas. O segundo nível é constituído pelos critérios de decisão:

- Resistência: a resistência do material a ser utilizado para a confecção da estrutura da cadeira de rodas é um aspecto importante tendo em vista a forma de uso. A independência do usuário está diretamente ligada à sua circulação em diferentes tipos de terrenos, sendo necessário então que o material utilizado atenda a essa necessidade. Segundo Mistarihi et al. (2020), Usma-Alvarez, Subic, Burton, \& Fuss (2010) e Gunawarman et al. (2005) a resistência do material é um dos principais requisitos para a confecção de cadeira de rodas.

- Densidade: Segundo Gunawarman et al. (2005) o peso da cadeira de rodas é um dos aspectos mais importantes para o usuário. A maior mobilidade do usuário da cadeira de rodas está diretamente ligada ao peso do equipamento, cadeiras de rodas com peso elevado trazem consequências negativas aos usuários, como maior fadiga e a longo prazo problemas de saúde. Nesse aspecto, a densidade do material a ser utilizado para a fabricação da cadeira de rodas, deve ser verificado. O estudo de Cowan, Nash, Collinger, Koontz, \& Boninger (2009) e Berger et al. (2012) analisam o impacto do peso na propulsão das cadeiras de rodas.

- Custo: esse critério representa um dos principais aspectos de influência na decisão da aquisição de uma cadeira de rodas, o custo da matéria-prima. Nwaoha e Ashiedu (2015) consideraram o custo como critério importante a ser considerado em projetos de cadeiras de rodas. Tento em vista esse mesmo aspecto, Kwarciak et al. (2005) analisaram o custo de três tipos de suspensão de cadeiras de rodas manuais.

- Processamento: existem vários materiais que podem atender a necessidade do usuário de cadeiras de rodas, embora o processamento de muitos desses materiais inviabilize a sua utilização, por isso é necessário a verificação dos aspectos relacionados aos equipamentos, pressão e temperatura a serem utilizados para o processamento dos materiais.

Outros critérios podem ser abordados conforme as necessidades específicas de cada tipo de cadeira de rodas.

O terceiro nível da estrutura hierárquica apresenta as alternativas de decisão, os materiais: Aço SGP (JIS G3452), Aço STKM11A (JIS G3444), Aço SUS304, Aço - A36, Alumínio A5056, Alumínio A6053T5, Alumínio A7003-T5, Alumínio 6061-T6, Alumínio 7075, Titânio Ti-6Al-4V. 
A estrutura hierárquica do processo decisório é apresentada na Figura 1.

Figura 1

Estrutura hierárquica do método AHP

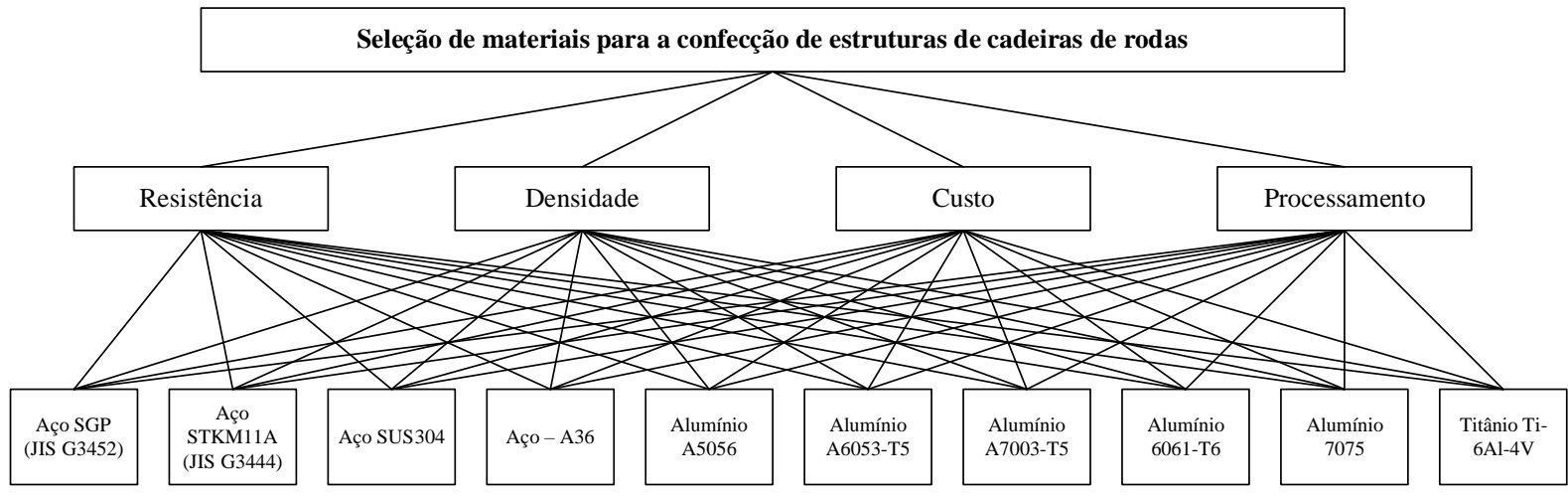

Fonte: Elaborado pelos autores.

\subsection{Mensuração dos critérios}

Para a aplicação do método AHP torna-se necessário a mensuração dos critérios quantificáveis, apresentando essas informações relativas a cada alternativa para que a tomada de decisão se torne mais precisa.

Na Tabela 1, são apresentadas as informações sobre os critérios relacionadas às alternativas.

Tabela 1

Informações sobre os critérios quantificáveis

\begin{tabular}{|c|c|c|c|}
\hline Materiais & Resistência (Mpa) & $\begin{array}{l}\text { Densidade } \\
\left(\mathrm{g} / \mathrm{cm}^{3}\right)\end{array}$ & $\begin{array}{l}\text { Custo } \\
(\$ / \mathrm{kg})\end{array}$ \\
\hline Aço SGP (JIS G3452) & 290 & 7,85 & 0,62 \\
\hline Aço STKM11A (JIS G3444) & 290 & 7,88 & 0,65 \\
\hline Aço SUS304 & 627 & 7,93 & 0,84 \\
\hline$A c ̧ o-A 36$ & 400 & 7,85 & 0,65 \\
\hline Alumínio A5056 & 290 & 2,64 & 5,41 \\
\hline Alumínio A6053-T5 & 185 & 2,69 & 3,75 \\
\hline Alumínio A7003-T5 & 360 & 2,79 & 2,1 \\
\hline Alumínio 6061-T6 & 310 & 2,71 & 5,16 \\
\hline Alumínio 7075-T6 & 572 & 2,80 & 2,15 \\
\hline Titânio Ti-6Al-4V & 950 & 4,43 & 28 \\
\hline
\end{tabular}

Fonte: Elaborado pelos autores. 
O critério processamento será analisado de forma qualitativa por especialistas da área.

\subsection{Aplicação do método AHP}

A aplicação do método AHP compreende: a coleta de dados para o desenvolvimento das matrizes de comparação, a análise da consistência dos julgamentos e a síntese dos resultados.

- Coleta de dados para o desenvolvimento das matrizes de comparação

A coleta de dados para a realização da comparação par a par entre os critérios e as alternativas se deu com especialistas da área, com base no conhecimento e experiências individuais. Os julgamentos comparativos consistem em determinar a importância relativa de cada elemento dentro de cada nível (Zafarani, Hassani, \& Bagherpour, 2014). A comparação par a par é realizada com base em uma escala numérica que varia de 1 a 9 ou da respectiva escala qualitativa (Saaty, 1990). Essa escala é empregada para comparar a importância dos pesos dos critérios, subcritérios e alternativas.

Com base nessa escala de importância, realiza-se o preenchimento das matrizes de comparação. O preenchimento é realizado por linhas, onde a diagonal principal é composta pelo valor 1, representando a comparação de um elemento por ele mesmo. Na linha 1 são inseridas as importâncias que os elementos dessa linha têm, em relação a cada elemento de todas as colunas e assim sucessivamente. Caso o elemento linha seja mais importante que elemento coluna, esse assume um valor de 2 a 9 dependendo do grau de importância. Caso contrário, se o elemento linha for menos importante ao elemento coluna, assume-se o grau de importância inverso, ou seja: $\frac{1}{2}$ a $\frac{1}{9}$. O resultado de cada comparação fornece uma matriz quadrada $(n \times n)$.

As comparações são realizadas visando encontrar o autovetor $w$ com o respectivo $\lambda_{\max }$ que satisfaz $A w=\lambda_{\max } w$, onde $\lambda_{\max }$ é o maior autovetor da matriz $A$. Então o autovetor $w$ com o respectivo $\lambda_{\max }$ é encontrado se $\left(A-\lambda_{\max } I\right) w=0$ (Çaliskan et al., 2013).

Esse procedimento se repete para todos os elementos em todos os níveis hierárquicos, de forma a sintetizar os vários vetores prioritários, esses vetores são ponderados com a prioridade global dos critérios principais e então são sintetizados (Zafarani et al., 2014).

- Análise de consistência dos julgamentos

A consistência é um indicador de que os julgamentos são coerentes. O método AHP fornece uma forma para medir a consistência da tomada de decisão e permite a revisão da decisão, a fim de se chegar a um nível aceitável de consistência (Arunraj \& Maiti, 2010).

O índice de consistência pode ser calculado através da Equação 1: 


$$
\mathrm{IC}=\left(\lambda_{\max ^{-}} n\right) /(n-1)
$$

Onde $\lambda_{\max }$ é o maior autovetor, e $n$ é o número de elementos ou a ordem da matriz $A$ (Mayyas et al., 2011).

Na aplicação do método AHP, a inconsistência é um fator mensurável e aceito tendo em vista a utilização de julgamentos humanos. Sendo assim, a inconsistência serve de alerta para os tomadores de decisão, e não é necessariamente um fator indesejável. Desta forma, a razão de consistência é calculada para verificar as inconsistências da decisão (Zafarani et al., 2014).

A razão de consistência $(C R)$ é a razão entre o índice de inconsistência IC e o índice de inconsistência aleatória (IR). Saaty (1986) propõe o cálculo da CR por meio da Equação 2:

$$
C R=I C / I R
$$

O IR é derivado de uma grande amostra de matrizes recíprocas geradas aleatoriamente utilizando a escala $1 / 9,1 / 8, \ldots, 1, \ldots, 8,9$ (Saaty, 1987).

Quanto maior for o CR, maior é a inconsistência. A inconsistência é aceitável desde que o valor do CR seja inferior a 0,10, sendo os valores de CR superiores a 0,10 os julgamentos não são considerados confiáveis, sendo necessária a revisão pelos decisores (Zafarani et al., 2014).

- Síntese dos resultados

Após a definição das prioridades e análise da consistência dos julgamentos o método AHP realiza a síntese dos resultados para a definição da alternativa de maior prioridade (Mayyas et al., 2011).

\section{Resultados e discussões}

Partindo da estrutura hierárquica do problema de decisão empregado no método AHP, para a seleção do material para a confecção da estrutura de cadeiras de rodas. No segundo nível hierárquico, foram definidas as prioridades dos critérios $(R=$ resistência; $D=$ densidade; $C=$ custo; $P=$ processamento) por meio da comparação par a par, formando assim a matriz de decisão, a qual foi então normalizada para a identificação dos vetores de preferência, conforme apresentado na Tabela 2. 
Tabela 2

Priorização dos critérios

Matriz de decisão

\begin{tabular}{|c|c|c|c|c|} 
& $\mathrm{R}$ & $\mathrm{D}$ & $\mathrm{C}$ & $\mathrm{P}$ \\
\hline $\mathrm{R}$ & 1 & $1 / 2$ & $1 / 3$ & 5 \\
\hline $\mathrm{D}$ & 2 & 1 & 2 & 5 \\
\hline $\mathrm{C}$ & 3 & $1 / 2$ & 1 & 5 \\
\hline $\mathrm{P}$ & $1 / 5$ & $1 / 5$ & $1 / 5$ & 1 \\
\hline
\end{tabular}

Fonte: Elaborado pelos autores.
Matriz normalizada

\begin{tabular}{c|c|c|c|c|} 
& R & D & C & P \\
\hline & 0,157 & 0,214 & 0,090 & 0,312 \\
\hline R & 0,315 & 0,428 & 0,545 & 0,312 \\
\hline D & 0,315 & 0,314 \\
\hline C & 0,473 & 0,214 & 0,272 & 0,312 \\
\hline P & 0,052 & 0,142 & 0,090 & 0,083 \\
\hline
\end{tabular}

Vetores de preferência

R $\quad 0,1939$

D $\quad 0,4006$

C 0,3183

P $\quad 0,0924$

Nessa análise, o critério densidade foi considerado o mais importante na seleção do material (40\%). Em ordem decrescente de prioridade, o custo do material apresenta-se na segunda colocação de prioridade $(31,8 \%)$, na terceira colocação o critério resistência $(19,3 \%)$ e, por último, o critério processamento do material $(9,2 \%)$.

A análise dos critérios gerou um índice de inconsistência de 0,08, estando a análise consistente de acordo com o método AHP, cujo índice máximo tolerado de inconsistência é 0,10 (Saaty, 1987).

Com base nas informações relativas aos pesos dos critérios, foram analisadas as alternativas de decisão: os possíveis materiais para a confecção de cadeiras de rodas. Nessa análise foram considerados 10 materiais, sendo quatro tipos de aço, cinco ligas de alumínio e um tipo de titânio, conforme apresentado na Tabela 1.

Na análise do terceiro nível da estrutura hierárquica do método AHP, a priorização referente a resistência, densidade e custo dos materiais analisados, os índices foram obtidos de forma direta por meio da normalização dos respectivos valores objetivos que foram apresentados na Tabela 1. 0 resultado da priorização é apresentado na Tabela 3. 
Tabela 3

Priorização das alternativas em relação aos critérios: resistência, densidade e custo

\begin{tabular}{lllll} 
& Materiais & Resistência & Densidade & Custo \\
\hline 1 & Aço SGP (JIS G3452) & 0,0678 & 0,0456 & 0,2149 \\
3 & $\begin{array}{l}\text { Aço STKM11A (JIS } \\
\text { G3444) }\end{array}$ & 0,0678 & 0,0455 & 0,2050 \\
4 & Aço SUS304 & 0,1467 & 0,0452 & 0,1586 \\
5 & Aço A36 & 0,0935 & 0,0456 & 0,2050 \\
6 & Alumínio A5056 & 0,0678 & 0,1358 & 0,0246 \\
7 & Alumínio A6053-T5 & 0,0432 & 0,1332 & 0,0355 \\
8 & Alumínio A7003-T5 & 0,0842 & 0,1285 & 0,0634 \\
9 & Alumínio 7075-T6 & 0,0725 & 0,1323 & 0,0258 \\
10 & $\quad$ Titânio Ti-6Al-4V & 0,1338 & 0,1280 & 0,0619
\end{tabular}

Fonte: Elaborado pelos autores.

Em relação ao critério processamento, a priorização das alternativas foi obtida por meio da comparação par a par entre as alternativas, posteriormente os dados foram normalizados para a obtenção dos vetores de preferência dos materiais, conforme apresentado na Tabela 4 e 5.

\section{Tabela 4}

Matriz de priorização das alternativas em relação ao critério: processamento

\begin{tabular}{|c|c|c|c|c|c|c|c|c|c|c|}
\hline & 1 & 2 & 3 & 4 & 5 & 6 & 7 & 8 & 9 & 10 \\
\hline 1 & 1 & 1 & 7 & 3 & $1 / 3$ & $1 / 7$ & 1 & 3 & $1 / 2$ & 7 \\
\hline 2 & 1 & 1 & 7 & 3 & $1 / 2$ & $1 / 5$ & 1 & $1 / 3$ & 2 & 7 \\
\hline 3 & $1 / 7$ & $1 / 7$ & 1 & $1 / 3$ & $1 / 7$ & $1 / 9$ & $1 / 3$ & $1 / 5$ & $1 / 4$ & 3 \\
\hline 4 & $1 / 3$ & $1 / 3$ & 3 & 1 & $1 / 5$ & $1 / 9$ & $1 / 5$ & $1 / 6$ & 2 & 5 \\
\hline 5 & 3 & 2 & 7 & 5 & 1 & $1 / 3$ & 3 & 1 & 5 & 9 \\
\hline 6 & 7 & 5 & 9 & 9 & 3 & 1 & 4 & 2 & 5 & 9 \\
\hline 7 & 1 & 1 & 3 & 5 & $1 / 3$ & $1 / 4$ & 1 & $1 / 3$ & 3 & 8 \\
\hline 8 & $1 / 3$ & 3 & 5 & 6 & 1 & $1 / 2$ & 3 & 1 & 5 & 9 \\
\hline 9 & 2 & $1 / 2$ & 4 & $1 / 2$ & $1 / 5$ & $1 / 5$ & $1 / 3$ & $1 / 5$ & 1 & 5 \\
\hline 10 & $1 / 7$ & $1 / 7$ & $1 / 3$ & $1 / 5$ & $1 / 9$ & $1 / 9$ & $1 / 8$ & $1 / 9$ & $1 / 5$ & 1 \\
\hline
\end{tabular}

Fonte: Elaborado pelos autores. 
Tabela 5

Matriz normalizada e vetores de preferência das alternativas em relação ao critério: processamento

Matriz normalizada

Vetores de

preferência

\begin{tabular}{|c|c|c|c|c|c|c|c|c|c|c|c|c|}
\hline & 1 & 2 & 3 & 4 & 5 & 6 & 7 & 8 & 9 & 10 & \\
\hline 1 & 0,063 & 0,072 & 0,151 & 0,091 & 0,049 & 0,048 & 0,071 & 0,360 & 0,021 & 0,111 & 1 & 0,103 \\
\hline 2 & 0,063 & 0,072 & 0,151 & 0,091 & 0,500 & 0,068 & 0,071 & 0,040 & 0,084 & 0,111 & 2 & 0,125 \\
\hline 3 & 0,009 & 0,010 & 0,022 & 0,010 & 0,143 & 0,038 & 0,024 & 0,024 & 0,010 & 0,048 & 3 & 0,033 \\
\hline 4 & 0,021 & 0,010 & 0,065 & 0,030 & 0,200 & 0,038 & 0,014 & 0,020 & 0,084 & 0,079 & 4 & 0,056 \\
\hline 5 & 0,188 & 0,144 & 0,151 & 0,151 & 1,000 & 0,113 & 0,214 & 0,120 & 0,209 & 0,143 & 5 & 0,243 \\
\hline 6 & 0,439 & 0,359 & 0,194 & 0,272 & 3,000 & 0,338 & 0,286 & 0,240 & 0,209 & 0,143 & 6 & 0,547 \\
\hline 7 & 0,063 & 0,072 & 0,065 & 0,151 & 0,333 & 0,084 & 0,071 & 0,040 & 0,125 & 0,127 & 7 & 0,113 \\
\hline 8 & 0,021 & 0,215 & 0,108 & 0,182 & 1,000 & 0,169 & 0,214 & 0,120 & 0,209 & 0,143 & 8 & 0,238 \\
\hline 9 & 0,125 & 0,036 & 0,086 & 0,015 & 0,200 & 0,068 & 0,024 & 0,024 & 0,042 & 0,079 & 9 & 0,069 \\
\hline 10 & 0,009 & 0,010 & 0,007 & 0,006 & 0,111 & 0,038 & 0,009 & 0,013 & 0,008 & 0,016 & 10 & 0,022 \\
\hline
\end{tabular}

Fonte: Elaborado pelos autores.

Posteriormente, os vetores de preferência das alternativas foram multiplicados pelo vetor de preferência dos critérios gerando assim o resultado final da análise, conforme apresentado na Tabela 6 .

Tabela 6

Ponderação final

Materiais

Resistência Densidade Custo Processamento

Peso dos

Peso final

Aço SGP (JIS G3452)
Aço STKM11A (JIS G3444)
Aço SUS304
Aço A36
Alumínio A5056
Alumínio A6053 - T5
Alumínio A7003 - T5
Alumínio 6061 - T6
Alumínio 7075 - T6
Titânio Ti - 6Al - 4V

$\left[\begin{array}{l}0,068 \\ 0,067 \\ 0,146 \\ 0,093 \\ 0,067 \\ 0,043 \\ 0,084 \\ 0,072 \\ 0,133 \\ 0,222\end{array}\right.$

0,045

0,214

0,045

0,214

0,045

0,158

$0,045 \quad 0,205$

$0,135 \quad 0,024$

$0,133 \quad 0,035$

$0,128 \quad 0,063$

$0,132 \quad 0,025$

$0,128 \quad 0,061$

Titânio Ti $-6 \mathrm{Al}-4 \mathrm{~V}$

0,080

0,004

$\left.\begin{array}{c}0,103 \\ 0,125 \\ 0,033 \\ 0,056 \\ 0,243 \\ 0,547 \\ 0,113 \\ 0,238 \\ 0,069 \\ 0,022\end{array}\right]$

critérios

Fonte: Elaborado pelos autores.

$\mathrm{x}\left[\begin{array}{l}0,193 \\ 0,400 \\ 0,318 \\ 0,092\end{array}\right]=\left[\begin{array}{l}0,109 \\ 0,108 \\ 0,100 \\ 0,106 \\ 0,097 \\ 0,123 \\ 0,098 \\ 0,097 \\ 0,103 \\ 0,079\end{array}\right]$

A ordenação dos resultados da ponderação, gerou a classificação final das alternativas de materiais para a confecção de cadeiras de rodas, conforme apresentado na Figura 2. 
Figura 2

Classificação final das alternativas

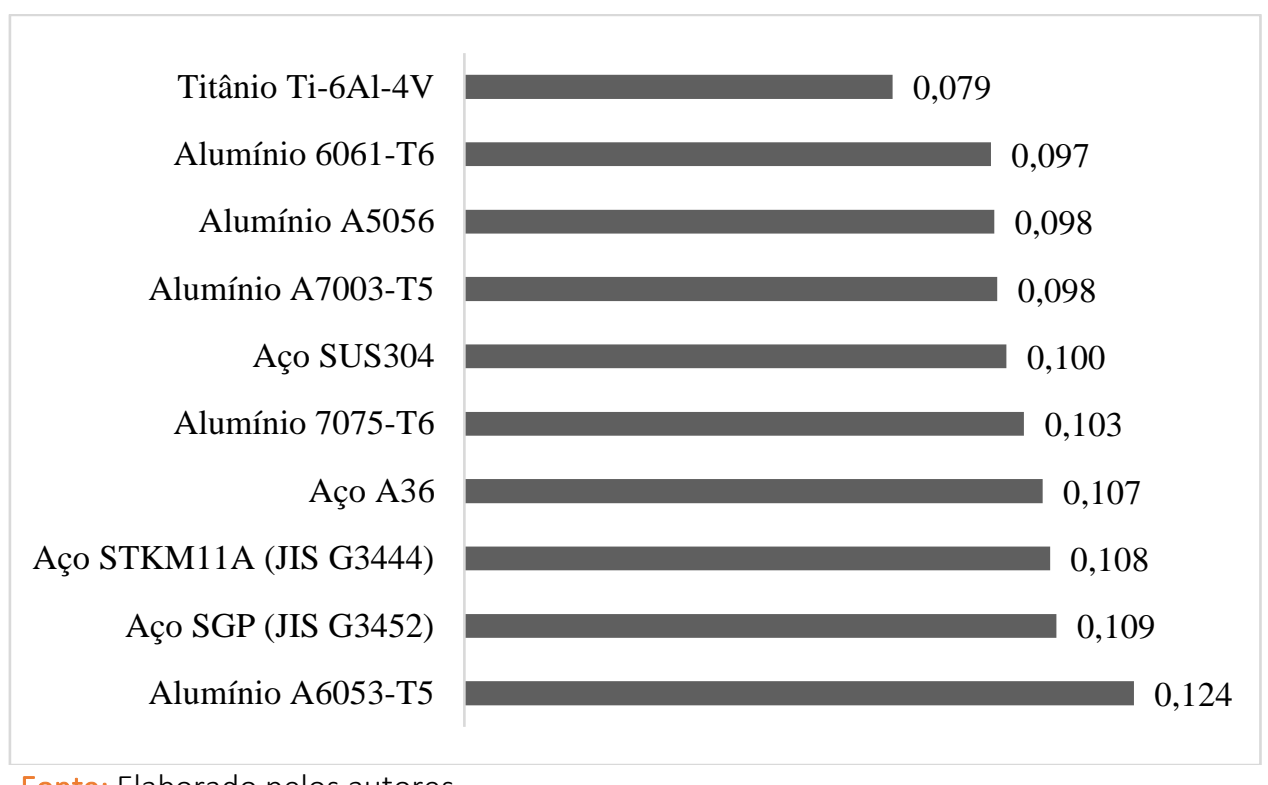

Fonte: Elaborado pelos autores.

De acordo com os resultados obtidos, definiu-se a liga de alumínio A6053-T5 como o melhor material para confecção da estrutura da cadeira de rodas, com 12,4\% de prioridade. Essa liga de alumínio é a menos densa entre os materiais analisados, indo assim de encontro com a priorização dos critérios, onde o critério menor densidade foi considerado o mais importante na análise. Esse resultado vai de encontro com o exposto por Berger, et al. (2012), Cowan, et al. (2009) e Gunawarman et al. (2005), que consideram o peso do material utilizado um dos aspectos mais importantes para a confecção de cadeiras de rodas.

Na sequência, classificaram-se os Aços: SGP (JIS G3452) com 10,9\%; STKM11A (JIS G3444) com 10,8\% e A36 com 10,7\%. Esses três materiais obtiveram uma classificação muito próxima por possuírem densidade semelhante e por serem os materiais mais baratos entres os analisados, sendo o critério custo o segundo mais importante na análise. Mistarihi et al. (2020) e Nwaoha \& Ashiedu (2015) igualmente identificaram que o custo foi o segundo fator mais importante para o projeto de cadeira de rodas. Porém, esses resultados divergem de estudos como de Rahman, Tahiduzzaman, \& Dey (2018) e Ahmad et al. (2017) que afirmam que o custo não possui grande importância para o projeto de cadeiras de rodas. Esses resultados divergem devido aos diferentes objetivos dos respectivos estudos.

Entre os materiais com classificações intermediárias encontram-se ainda a liga de alumínio 7075-T6 com 10,3\%, Aço SUS304 com 10\%, as ligas de alumínio A7003-T5 e A5056, ambas com 9,8\% de prioridade na decisão, seguido do alumínio 6061-T6 com 9,7\%.

O Titânio Ti-6Al-4V foi classificado em último lugar na análise com 7,9\% de prioridade na decisão. Esse que é o material mais resistente entre os analisados e possui densidade intermediária, 
porém o seu custo é o mais elevado de todos, com diferenças exorbitantes perante aos demais materiais, inviabilizando a sua utilização comercial. Mistarihi et al. (2020) e Usma-Alvarez et al. (2010) porém, consideram a resistência do material como o principal aspecto a ser considerado na escolha do material para a confecção de cadeiras de rodas.

Na classificação dos materiais, é possível verificar que a relação densidade $x$ custo do material predominou na classificação geral, já que esses foram os critérios mais importantes, como pode ser verificado na análise anterior em relação ao segundo nível da estrutura hierárquica de decisão.

Essa análise gerou um índice de inconsistência de 0,09, muito próximo ao índice tolerado pelo método AHP de 0,10. Esse fato deve-se ao número elevado de alternativas, pois quanto maior a estrutura hierárquica, maior o número de análises, o que consequentemente acaba influenciando o raciocínio humano na tomada de decisão.

Pode-se verificar na classificação geral, que os materiais se mantiveram em posições muito próximas, com variações decimais. Isso significa que a análise possui uma sensibilidade muito alta, onde pequenas variações entre os índices alteraria a classificação dos materiais analisados.

Segundo Mistarihi et al. (2020) e Usma-Alvarez et al. (2010) a escolha do material adequado para a confecção da cadeira de rodas, é um dos principais fatores a ser considerado no projeto de cadeira de rodas. O diferencial desse estudo está na aplicação do método multicritério de tomada de decisão AHP para a seleção dos materiais em projetos de cadeiras de rodas. Conforme exposto por Nwaoha e Ashiedu (2015) o método AHP é uma ferramenta viável para a estimativa de peso dos critérios e seleção de alternativas no design da cadeira de rodas. O método AHP foi utilizado visando inserir a experiência de especialistas na tomada de decisão para a seleção dos materiais para o projeto de cadeira de rodas, além dos aspectos técnicos dos materiais.

O método AHP não é comumente empregado em projetos de desenvolvimento de cadeira de rodas, porém é comum a existência de aspectos objetivos e subjetivos nesse tipo de tomada de decisão. Conforme exposto por Yuan \& Guan (2014) os métodos tradicionais de projetos de cadeira de rodas nem sempre conseguem atender a certas especificidades e aspectos subjetivos na tomada de decisão. A falha na identificação de critérios importantes pode resultar em erros de projeto, fabricação, montagem e operação (Nwaoha \& Ashiedu, 2015). Para tanto, o método AHP contribui em relação a esses aspectos.

Os estudos Mistarihi et al. (2020), Ahmad et al. (2017), Nwaoha e Ashiedu (2015) e Yuan e Guan (2014) aplicam as técnicas de decisão multicritério em projetos de cadeira de rodas, porém relacionados a diferentes fases e características do projeto de cadeiras de rodas. Esses estudos demonstram que os métodos multicritérios podem ser utilizados em vários contextos de tomada de decisão e auxiliar no desenvolvimento das várias etapas de um projeto de cadeira de rodas. 
Conclusões

Uma metodologia com base na representação hierárquica do método AHP foi proposta para auxiliar o desenvolvimento de projetos de cadeiras de rodas, por meio da seleção de materiais para a confecção da estrutura de cadeiras de rodas, visando atender as necessidades dos usuários que procuram equipamentos leves, resistentes à um custo acessível.

$\mathrm{Na}$ análise proposta para a validação do método, identificou-se que a densidade (peso) e custo são os fatores mais importantes na escolha do material para a confecção da estrutura da cadeira de rodas. E atendendo a esses requisitos, bem como os demais critérios analisados, a liga de alumínio A7075-T6 foi considerada a alternativa mais satisfatória de acordo com os parâmetros e seus respectivos pesos, sendo esse considerado o melhor material para a confecção de estruturas de cadeiras de rodas, entre os materiais analisados. As respostas foram obtidas com base nas ponderações efetuadas entre a importância dos critérios e as informações quantitativas e qualitativas das alternativas.

Embora o alumínio não seja um dos materiais mais baratos, perdendo essa posição para o aço, o alumínio é um dos materiais mais leves, sendo esse requisito representado pela densidade na análise, a qual obteve o maior índice na priorização dos critérios. É também um material de fácil processamento o que torna o seu custo final acessível.

O presente estudo busca trazer contribuições teóricas relacionadas a uma nova aplicabilidade do método multicritério de tomada de decisão AHP. E contribuições práticas, acerca da utilização do método AHP para a seleção dos materiais em projetos de cadeiras de rodas, bem como, demonstra a possibilidade de aplicação do método em diferentes projetos de desenvolvimento de produtos, onde existam fatores subjetivos que possam implicar o processo decisório.

A abordagem desse estudo partiu da análise técnica e conceitual, tendo como limitação o desenvolvimento do protótipo da cadeira de rodas para testes específicos de desempenho. Sendo assim, sugere-se como estudos futuros a aplicação de outras técnicas de decisão multicritério, bem como o desenvolvimento de protótipos e testes específicos para se chegar a conclusões decisivas, afim de alcançar a melhor qualidade no projeto da cadeira de rodas.

\section{Agradecimentos}

A Coordenação de Aperfeiçoamento de Pessoal de Nível Superior (CAPES) pela concessão de bolsa de estudo para a realização da pesquisa. 


\section{Referências}

Ahmad, M. N., Maidin, N. A., Rahman, M. H. A., \& Osman, M. H. (2017). Conceptual Design Selection of Manual Wheelchair for Elderly by Analytical Hierarchy Process (AHP) Method: A Case Study. International Journal of Applied Engineering Research, 12, 6710-6719. Disponível em: https://www.ripublication.com/ijaer17/ijaerv12n17_58.pdf

Arunraj, N. S., \& Maiti, J. (2010). Risk-based maintenance policy selection using AHP and goal programming. Safety Science, 48, 238-247. DOI: https://doi.org/10.1016/j.ssci.2009.09.005

Banville, M., Landry, M., Martel, J. M., \& Boulaire, C. (1998). A stakeholver approach to MCDA. System Research and Behavioral Science, 15(1), 502-519. DOI: https://doi.org/10.1002/(SICI)10991743(199801/02)15:1<15::AID-SRES179>3.0.CO;2-B

Berger, M. A. M., Van Nieuwenhuizen, M., Van Der Ent, M., \& Van Der Zande, M. (2012). Development of a new wheelchair for wheelchair basketball players in the Netherlands. Procedia Engineering, 34, 331-336. DOI: https://doi.org/10.1016/j.proeng.2012.04.057

Çaliskan, H., Kursuncu, B., Kurbanoglu, C., \& Güven, S. Y. (2013). Material selection for the tool holder working under hard milling conditions using different multi criteria decision making methods. Materials and Design, 45, 473-479. DOI: https://doi.org/10.1016/j.matdes.2012.09.042

Cowan, R. E., Nash, M. S., Collinger, J. L., Koontz, A. M., \& Boninger, M. L. (2009). Impact of surface type, wheelchair weight, and axle position on wheelchair propulsion by novice older adults. Archives of Physical Medicine and Rehabilitation, 90, 1076-1083. DOI: https://doi.org/10.1016/j.apmr.2008.10.034

Fitzgerald, S., Cooper, R. A., Boninger, M. L., \& Rentschler, A. J. (2001). Comparison of fatigue live for 3 types of manual wheelchairs. Archives of Physical Medicine and Rehabilitation, 82, 1484-1488. DOI: https://doi.org/10.1053/apmr.2001.26139

Gunawarman, B., Niinomi, M., Akahori, T., Souma, T., Ikeda, M., \& Toda, H. (2005). Mechanical properties and microstructures of low cost $\beta$ titanium alloys for healthcare applications. Materials Science and Engineering C, 25, 304-311. DOI: https://doi.org/10.1016/j.msec.2004.12.015

Hybois, S., Puchaud, P., Bourgain, M., Lombart, A., Bascou, J., Lavaste, F., Fodé, P., Pillet, H., \& Sauret, C. (2019). Comparison of shoulder kinematic chain models and their influence on kinematics and kinetics in the study of manual wheelchair propulsion. Medical Engineering and Physics, 69, 153-160. DOI: https://doi.org/10.1016/j.medengphy.2019.06.002

Koontz, A. M., Brindle, E. D., Kankipati, P., Feathers, D., \& Cooper, R. A. (2010). Design features that affect the maneuverability of wheelchairs and scooters. Archives of Physical Medicine and Rehabilitation, 91, 759-764. DOI: https://doi.org/10.1016/j.apmr.2010.01.009

Kwarciak, A. M., Cooper, R. A., Ammer, W. A., Fitzgerald, S., Boninger, M. L., \& Cooper, R. (2005). Fatigue testing of selected suspension manual wheelchairs using ANSI/RESNA satandards. Archives of Physical Medicine and Rehabilitation, 86, 123-129. DOI: https://doi.org/10.1016/j.apmr.2003.11.038

Mayyas, B., Shen, Q., Mayyas, A., Abdelhamid, M., Shan, D., Qattawi, A., \& Omar, M. (2011). Using quality function deployment and analytical hierarchy process for material selection of body-in- 
white. Materials and Design, 32, 2771-2782. DOI:

https://doi.org/10.1016/j.matdes.2011.01.001

Mistarihi, M. Z., Okour, R. A., \& Mumani, A. A. (2020). An integration of a QFD model with Fuzzy-ANP approach for determining the importance weights for engineering characteristics of the proposed wheelchair design. Applied Soft Computing Journal, 90, 1-12. DOI: https://doi.org/10.1016/j.asoc.2020.106136

Mohammadshahi, Y. (2013). A state-of-art survey on TQM applications using MCDM techniques. Decision Science Letters, 2, 125 - 134. DOI: https://doi.org/10.5267/j.dsl.2013.03.004

Nwaoha, T. C., \& Ashiedu, F. I. (2015). Engineering Judgment in Wheelchair Design Criteria: An Analytical Hierarchy Process (AHP) Approach. Journal of Sustainable Technology, 6, $32-42$. Disponível em: https://www.futa.edu.ng/journal/home/paperd/340/31/10

Quaglia, G., Franco, W., \& Oderio, R. (2011). Wheelchair.q, a motorized wheelchair with stair climbing ability. Mechanism and Machine Theory, 46, 1601-1609. DOI: https://doi.org/10.1016/j.mechmachtheory.2011.07.005

Rahman, M., Tahiduzzaman, M., \& Dey, S. K. (2018). QFD Based Product Design and Development of Weight Measuring Chair for the Benefits of Physically Challenged Person. American Journal of Industrial Engineering, 5, 12-16. DOI: https://doi.org/10.12691/ajie-5-1-2

Ravenek, K. E., Ravenek, M. J., Hitzig, S. L., \& Wolfe, D. L. (2012). Assessing quality of life in relation to physical activity participation in persons with spinal cord injury: A systematic review. Disability and Health Journal, 5, 213-223. DOI: https://doi.org/10.1016/j.dhjo.2012.05.005

Roy, B., \& Vanderpooten, D. (1996). The European School of MCDA: Emergence, Basic Features and Current Works. Journal of Multicriteria Decision Analysis, 5, 22-38. DOI: https://doi.org/10.1002/(SICI)1099-1360(199603)5:1<22::AID-MCDA93>3.0.CO;2-F

Ruíz-Serrano, A., Posada-Gómez, R., Sibaja, A. M., Rodríguez, G. A., Gonzalez-Sanchez, B. E., \& Sandoval-Gonzalez, O. O. (2013). Development of a dual control system applied to a smart wheelchair, using magnetic and speech control. Procedia Technology, 7, 158-165. DOI: https://doi.org/10.1016/j.protcy.2013.04.020

Saaty, R. W. (1987). The analytic hierarchy process - what it is and how it is used. Mathematical Modeling, 9(3-5), 161-176. DOI: https://doi.org/10.1016/0270-0255(87)90473-8

Saaty, T. L. (1990). How to make a decision: The analytic hierarchy process. European Journal of Operational Research, 48, 9-26. DOI: https://doi.org/10.1016/0377-2217(90)90057-I

Saaty, T. L. (1986). Axiomatic Foundation of the Analytic Hierarchy Process. Management Science, 32(7), 841-855. DOI: https://doi.org/10.1287/mnsc.32.7.841

Sprigle, S. (2009). On Impact of surface type, wheelchair weight, anda position on wheelchair propulsion by novice older adults. Archives of Physical Medicine and Rehabilitation, 90, 10731075. DOI: https://doi.org/10.1016/j.apmr.2009.04.002

Usma-Alvarez, C. C., Subic, A., Burton, M., \& Fuss, F. K. (2010). Identification of design requiriments for rugby wheelchairs using the QFD method. Procedia Engineering, 2, 2749-2755. DOI: https://doi.org/10.1016/j.proeng.2010.04.061 
Vaidya, O.S., \& Kumar, S. (2006). Analytic hierarchy process: An overview of applications. European Journal of Operational Research, 169, 1-29. DOI: https://doi.org/10.1016/j.ejor.2004.04.028

Ward, A. L., Sanjak, M., Duffy, K., Bravver, E., Williams, N., Nichols, M., \& Brooks, B. R. (2010). Power pheelchair prescription, utilization, satisfaction, and cost for patients with amyotrophic lateral sclerosis: preliminary data for evidence-based guidelines. Archives of Physical Medicine and Rehabilitation, 91, 268-272. DOI: https://doi.org/10.1016/j.apmr.2009.10.023

Yuan, Y., \& Guan, T. (2014). Design of Individualized Wheelchairs Using AHP and Kano Model. Advances in Mechanical Engineering, 14, 1-6. DOI: https://doi.org/10.1155/2014/242034

Zafarini, H. R., Hassani, A., \& Bagherpour, E. (2014). Achieving a desirable combination of strength and workability in $\mathrm{Al} / \mathrm{SiC}$ composites by AHP selection method. Journal of Alloys and Compounds, 589, 295-300. DOI: https://doi.org/10.1016/j.jallcom.2013.11.181 